\title{
含苯并咪唑环二酰肼衍生物的合成与结构表征
}

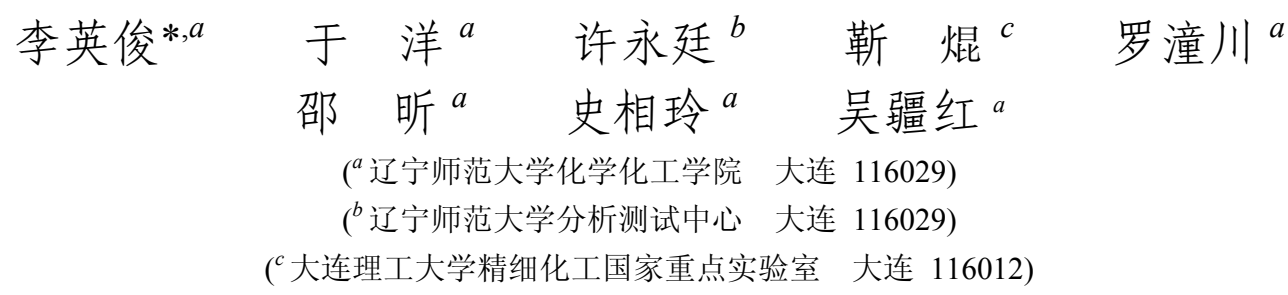

\begin{abstract}
摘要 以酚和氯乙酸为初始原料, 经一系列反应, 合成出了 10 个新型的含苯并咪唑环二酰肼衍生物 $7 \mathbf{a} \sim 7 \mathbf{j}$. 利用 ${ }^{1} \mathrm{H}$ NMR, ${ }^{13} \mathrm{C}$ NMR, 2D NMR(包括 HMBC, HSQC,${ }^{1} \mathrm{H}-{ }^{1} \mathrm{H}$ COSY 和 NOESY) 和变温 ${ }^{1} \mathrm{H}$ NMR 技术对代表化合物 7c 进行了 ${ }^{1} \mathrm{H}$ 和 ${ }^{13} \mathrm{C}$ NMR 谱归属并确定了其空间结构, 证明了其在室温及 DMSO 中存在着 $\mathbf{A}$ 和 $\mathbf{B}$ 两种互变异构体, 并且 $\mathbf{A}$ 异构体 占优势(87.65\%). 据此, 对其它目标化合物的 ${ }^{1} \mathrm{H}$ NMR 也进行了归属, A 异构体含量为 $80.55 \% \sim 89.90 \%$.
\end{abstract} 关键词 二酰肼; 合成; 表征

\section{Synthesis and Characterization of Dihydrazide Derivatives Containing Benzimidazole Ring}

\author{
Li, Yingjun $^{*, a} \quad$ Yu, Yang $^{a} \quad \mathrm{Xu}$, Yongting $^{b} \quad$ Jin, Kun $^{c} \quad$ Luo, Tongchuan $^{a}$ \\ Shao, $\mathrm{Xin}^{a} \quad$ Shi, Xiangling ${ }^{a} \quad \mathrm{Wu}$, Jianghong $^{a}$ \\ $\left({ }^{a}\right.$ College of Chemistry and Chemical Engineering, Liaoning Normal University, Dalian 116029) \\ ( ${ }^{b}$ Analysis and Inspection Center, Liaoning Normal University, Dalian 116029) \\ ( ${ }^{c}$ State Key Laboratory of Fine Chemicals, Dalian University of Technology, Dalian 116012)
}

\begin{abstract}
Ten novel dihydrazide derivatives containing benzimidazole ring (7a $\sim \mathbf{7 j}$ ) were synthesized by (un)substituted phenol and chloroacetic acid as starting materials via a series of reactions. The structure of representative compound $7 \mathbf{c}$ was confirmed by ${ }^{1} \mathrm{H}$ NMR, ${ }^{13} \mathrm{C}$ NMR, 2D NMR $\left({ }^{1} \mathrm{H}-{ }^{1} \mathrm{H}\right.$ COSY, HSQC, HMBC and NOESY) and variable-temperature ${ }^{1} \mathrm{H}$ NMR spectra. The experimental results indicate that compound 7c in DMSO at room temperature exists in the tautomeric $\mathbf{A}$ and $\mathbf{B}$ isomers, and $\mathbf{A}$ isomer is dominant (87.65\%). On the basis of the analytic results of compound $7 \mathbf{c},{ }^{1} \mathrm{H}$ NMR spectra of other target compounds were also assigned and the content of dominant $\mathbf{A}$ isomer is in the range of $80.55 \% \sim 89.90 \%$.
\end{abstract}

Keywords dihydrazide; synthesis; characterization

苯并咪唑(BZM)衍生物是构成许多生物活性分子 的重要骨架, 这类化合物因其广谱的生物活性和临床应 用已引起了科学工作者的关注. 例如, 苯并咪唑衍生物 具有抗癌 ${ }^{[1 \sim 3]} 、$ 抗炎 ${ }^{[4]}$ 、抗菌 ${ }^{[5]}$ 、杀虫 ${ }^{[6]}$ 等多种生物活性. 为了增强其药理学活性, 苯并咪唑环的各个位置均可被 取代, 如 2-取代的苯并咪唑类化合物多菌灵是潜在的抗 癌试剂. 近年来, 文献报道显示含有二酰朋单元的化合

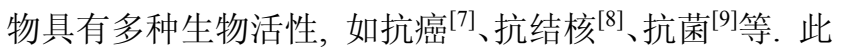
外, 在农业方面可作为杀虫剂 ${ }^{[10]}$, 在材料方面可作为液
晶材料 ${ }^{[11]}$.

为了寻求并笁选出更多具有应用前景的生物活性 分子, 本课题组在前期工作 ${ }^{[3,12,13]}$ 的基础上，设计将活 性基团苯并咪唑及二酰肼聚集在同一分子中合成出标 题化合物. 本文以酚和氯乙酸为初始原料, 经一系列反 应，合成出了 10 个未见文献报道的含苯并咪唑环二酰 肼衍生物 7a $\sim 7 \mathbf{j}$. 利用 IR, ${ }^{1} \mathrm{H}$ NMR, 元素分析对新化合 物 7a $\sim \mathbf{j}$ 的结构进行了表征. 通过对代表目标化合物 $7 \mathbf{c}$ 的 NMR 谱的研究, 确定了目标化合物的空间结构,

\footnotetext{
* E-mail: chemlab.lnnu@163.com

Received January 4, 2013; revised February 26, 2013; published online March 7, 2013.

Project supported by the Natural Science Foundation of Liaoning Province (No. 20102126).

辽宁省自然科学基金(No. 20102126)资助项目.
} 
证明了目标化合物在室温、DMSO 中存在着两种异构体 $\mathrm{A}$ 和 $\mathrm{B}$, 其中 $\mathrm{A}$ 异构体占优势. 目标化合物的药理活性 研究正在进行之中. 目标化合物的合成路线见 Scheme 1.

\section{1 实验部分}

\section{1 仪器和试剂}

仪器: X-5 显微熔点测定仪(温度计未经校正); 德国 TENSOR 27 型FT-IR 红外光谱仪, WGH-30 型双光束红 外分光光度计, 液膜或 $\mathrm{KBr}$ 压片; 德国 BRUKER 500 $\mathrm{MHz}$ 核磁共振仪, 美国 Varian INOVA $400 \mathrm{MHz}$ 核磁共 振仪, DMSO- $d_{6}$ 为溶剂, TMS 为内标; 德国 Elementar 公 司 Vario EL 型元素分析仪; 所用试剂均为分析纯.

\section{2 中间体的合成}

芳氧乙酸(1)、2-芳氧甲基苯并咪坐(2)、2-芳氧甲基 苯并咪唑-1-乙酸乙酯(3)和 2-芳氧甲基苯并咪唑-1-乙酰 肼(4)按照参考文献[3]合成. 4-茮氧基苯甲酸(5)按照参 考文献[14]合成.4-芐氧基苯甲酰氯(6)按照参考文献 [15]方法合成.

\section{3 目标化合物 $7 \mathrm{a} \sim 7 \mathrm{j}$ 的合成通法}

向 $100 \mathrm{~mL}$ 干燥的雉形瓶中依次加入 $0.21 \mathrm{~g}(0.85$ mmol）4-苠氧基苯甲酰氯(6), $15 \mathrm{~mL} \mathrm{CH}_{2} \mathrm{Cl}_{2}, 0.69 \mathrm{mmol}$ 2-芳氧甲基苯并咪唑-1-乙酰肼(4), 0.07 g PEG-400, $0.028 \mathrm{~g}(0.69 \mathrm{mmol}) \mathrm{NaOH}(\mathrm{s})$ 和 $10 \mathrm{~mL} \mathrm{H} \mathrm{H}_{2} \mathrm{O}$, 室温搅拌 5 h. 反应结束后, 水浴蒸出溶剂 $\mathrm{CH}_{2} \mathrm{Cl}_{2}$, 抽滤得粗产品. $\mathrm{DMF}$ 重结晶后得到目标化合物 $\mathbf{7} \mathbf{a} \sim \mathbf{7 j}$. 其物理常数及 波谱数据见表 $1 \sim 4$.

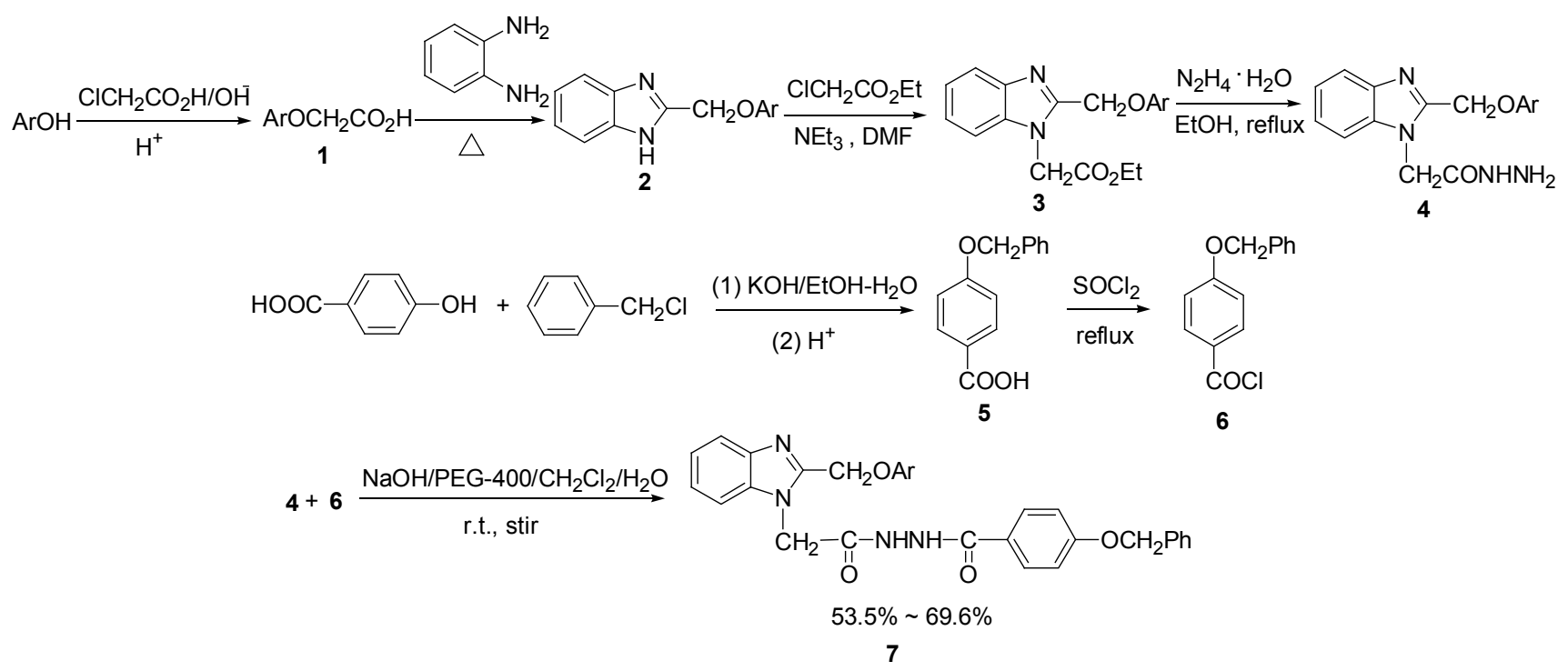

$\mathrm{Ar}=\mathrm{C}_{6} \mathrm{H}_{5}(\mathbf{a}), 3-\mathrm{CH}_{3} \mathrm{C}_{6} \mathrm{H}_{4}(\mathbf{b}), 4-\mathrm{CH}_{3} \mathrm{C}_{6} \mathrm{H}_{4}(\mathbf{c}), 2,4-\left(\mathrm{CH}_{3}\right)_{2} \mathrm{C}_{6} \mathrm{H}_{3}(\mathbf{d}), 2-\mathrm{CH}_{3} \mathrm{OC}_{6} \mathrm{H}_{4}(\mathbf{e}), 4-\mathrm{CH}_{3} \mathrm{OC}_{6} \mathrm{H}_{4}(\mathbf{f}), 4-\mathrm{IC}_{6} \mathrm{H}_{4}(\mathbf{g}), 4-\mathrm{ClC}_{6} \mathrm{H}_{4}(\mathbf{h}), 1-\mathrm{C}_{10} \mathrm{H}_{7}(\mathbf{i})$, $2-\mathrm{C}_{10} \mathrm{H}_{7}(\mathbf{j})$

\section{Scheme 1}

表 1 目标化合物 $7 \mathbf{a} \sim 7 \mathbf{j}$ 的物理性质及元素分析数据

Table 1 Physical properties and elemental analyses of target compounds $7 \mathbf{a} \sim 7 \mathbf{j}$

\begin{tabular}{|c|c|c|c|c|c|c|}
\hline \multirow{2}{*}{ Compd. } & \multirow{2}{*}{ Color } & \multirow{2}{*}{ m.p. $/{ }^{\circ} \mathrm{C}$} & \multirow{2}{*}{ Yield $/ \%$} & \multicolumn{3}{|c|}{ Elemental analysis, found (calcd) } \\
\hline & & & & $\mathrm{C}$ & $\mathrm{H}$ & $\mathrm{N}$ \\
\hline $7 \mathbf{a}$ & 白色固体粉末 & $242.5 \sim 243.0$ & 64.4 & $71.36(71.13)$ & $5.05(5.17)$ & $10.98(11.06)$ \\
\hline $7 \mathbf{b}$ & 白色固体粉末 & $239.9 \sim 240.3$ & 61.3 & $71.31(71.52)$ & $5.52(5.42)$ & $10.56(10.76)$ \\
\hline $7 \mathrm{c}$ & 白色固体粉末 & $224.1 \sim 225.1$ & 69.6 & $71.35(71.52)$ & $5.28(5.42)$ & $10.89(10.76)$ \\
\hline 7d & 白色固体粉末 & $247.2 \sim 247.7$ & 60.1 & $72.14(71.89)$ & $5.57(5.66)$ & $10.58(10.48)$ \\
\hline $7 e$ & 白色固体粉末 & $236.9 \sim 237.4$ & 53.5 & $69.61(69.39)$ & $5.43(5.26)$ & $10.29(10.44)$ \\
\hline $7 f$ & 白色固体粉末 & $224.6 \sim 226.1$ & 57.2 & $69.23(69.39)$ & $5.39(5.26)$ & $10.23(10.44)$ \\
\hline $7 g$ & 白色固体粉末 & $243.5 \sim 244.1$ & 59.7 & $57.11(56.97)$ & $4.17(3.98)$ & $9.03(8.86)$ \\
\hline $7 \mathrm{~h}$ & 白色固体粉末 & $230.1 \sim 231.3$ & 61.7 & $66.37(66.60)$ & $4.81(4.66)$ & $10.18(10.36)$ \\
\hline $7 \mathbf{i}$ & 白色固体粉末 & $284.4 \sim 284.7$ & 65.1 & $73.22(73.37)$ & $4.95(5.07)$ & $9.98(10.07)$ \\
\hline $7 \mathbf{j}$ & 白色固体粉末 & $248.0 \sim 249.5$ & 68.5 & $73.47(73.37)$ & $5.28(5.07)$ & $9.91(10.07)$ \\
\hline
\end{tabular}


表 2 目标化合物 $7 \mathbf{a} \sim 7 \mathbf{j}$ 的 IR 数据

Table 2 IR data of target compounds $7 \mathbf{a} \sim 7 \mathbf{j}$

\begin{tabular}{cl}
\hline Compd. & \multicolumn{1}{c}{$\mathrm{IR}(\mathrm{KBr}) v / \mathrm{cm}^{-1}$} \\
\hline $\mathbf{7 a}$ & $3176,3040,2944,2866,1685,1570,1496,1468,1606,1510,1439,1379,1243,1015$ \\
$\mathbf{7 b}$ & $3198,3064,2926,2853,1685,1609,1572,1483,1467,1378,1248,1021$ \\
$\mathbf{7 c}$ & $3236,3036,2928,2864,1684,1608,1576,1512,1468,1382,1244,1022$ \\
$\mathbf{7 d}$ & $3188,3034,2921,2855,1676,1604,1571,1504,1463,1437,1377,1251,1024$ \\
$\mathbf{7}$ & $3172,3037,2941,2873,1681,1571,1464,1606,1506,1435,1379,1254,1019$ \\
$\mathbf{7}$ & $3175,3040,2926,2856,1682,1605,1572,1507,1464,1437,1381,1237,1025$ \\
$\mathbf{7 g}$ & $3236,3061,2940,2855,1677,1610,1571,1517,1484,1463,1377,1244,1031$ \\
$\mathbf{7 h}$ & $3231,3063,2941,2853,1677,1610,1573,1512,1485,1468,1377,1241,1032$ \\
$\mathbf{7} \mathbf{7}$ & $3233,3054,2936,2865,1672,1608,1571,1511,1474,1372,1244,1021$ \\
$\mathbf{7 j}$ & $3196,3057,2925,2854,1683,1605,1575,1511,1467,1386,1254,1014$ \\
\hline
\end{tabular}

表 3 化合物 $7 \mathbf{c}$ 的 ${ }^{1} \mathrm{H} \mathrm{NMR}$ 和 ${ }^{13} \mathrm{C}$ NMR 数据(DMSO- $\left.d_{6}, 500 \mathrm{MHz}\right)$

Table $3{ }^{1} \mathrm{H}$ NMR and ${ }^{13} \mathrm{C}$ NMR spectral data of compound $7 \mathrm{c}\left(\mathrm{DMSO}-d_{6}, 500 \mathrm{MHz}\right)$

\begin{tabular}{|c|c|c|c|c|c|c|c|c|}
\hline \multirow{2}{*}{ No. } & \multicolumn{2}{|l|}{$\delta_{\mathrm{H}}$} & \multicolumn{2}{|c|}{$\delta_{\mathrm{C}}$} & \multirow{2}{*}{$\mathrm{HMBC}^{b}$} & \multirow{2}{*}{ HSQC } & \multirow{2}{*}{${ }^{1} \mathrm{H}-{ }^{1} \mathrm{HCOSY}$} & \multirow{2}{*}{ NOESY } \\
\hline & $\mathbf{A}$ & B & $\mathbf{A}$ & B & & & & \\
\hline 1 & 1 & 1 & 1 & 1 & 1 & l & l & 1 \\
\hline 2 & / & l & 150.1 & l & l & l & l & l \\
\hline 3 & / & l & / & / & / & / & / & l \\
\hline 4 & / & / & 141.8 & - & l & / & / & / \\
\hline 5 & $7.65(\mathrm{~d}, J=7.9 \mathrm{~Hz})$ & - & 119.3 & - & C-7, C-9 & + & H-6 & / \\
\hline 6 & $7.23(\mathrm{t}, J=7.4 \mathrm{~Hz})$ & - & 121.9 & - & C- $4, C-8$ & + & $\mathrm{H}-5, \mathrm{H}-7$ & / \\
\hline 7 & $7.29(\mathrm{t}, J=7.7 \mathrm{~Hz})$ & - & 122.8 & - & C-5, C-9 & + & H-6, H-8 & / \\
\hline 8 & $7.57(\mathrm{~d}, J=8.0 \mathrm{~Hz})$ & - & 110.4 & - & C-4, C-6 & + & $\mathrm{H}-7$ & / \\
\hline 9 & / & / & 135.9 & - & 1 & 1 & / & / \\
\hline $10 / 10^{\prime}$ & 5.38 & 5.27 & 62.8 & - & $\mathrm{C}-2, \mathrm{C}-29$ & + & / & H-30,34 \\
\hline $11 / 11^{\prime}$ & 5.15 & 5.22 & 69.4 & - & C-2, C-9, C-12 & + & l & H-13 \\
\hline 12 & / & / & 166.1 & - & / & / & / & I \\
\hline 13 & 10.47 & 9.69 & / & / & C-12 & l & $\mathrm{H}-14$ & H-11, H-14 \\
\hline 14 & 10.36 & 10.62 & / & / & C-15 & l & $\mathrm{H}-13$ & H-13, H-21 \\
\hline 15 & 1 & / & 164.9 & - & 1 & l & I & 1 \\
\hline 16 & / & / & 124.5 & 124.3 & / & l & l & 1 \\
\hline $17 / 17^{\prime}$ & $7.85(\mathrm{~d}, J=8.7 \mathrm{~Hz})$ & 7.90 & 129.4 & 129.6 & C-15, C-19 & + & H-18,20 & $\mathrm{H}-14$ \\
\hline $18 / 18^{\prime}$ & $7.10(\mathrm{~d}, J=8.3 \mathrm{~Hz})$ & 7.15 & 114.5 & 114.7 & C-16, C-19 & + & H-17,21 & $\mathrm{H}-22$ \\
\hline 19 & l & 1 & 161.1 & - & / & / & l & I \\
\hline $22 / 22^{\prime}$ & 5.17 & 5.11 & 44.6 & 43.6 & $\begin{array}{l}\text { C-19, C-23 } \\
\text { C- } 24,28\end{array}$ & + & / & H-18, H-24 \\
\hline 23 & I & / & 136.6 & - & / & l & / & I \\
\hline 24,28 & $7.45(\mathrm{~d}, J=7.4 \mathrm{~Hz})$ & - & 127.7 & - & $\mathrm{C}-22$ & + & - & $\mathrm{H}-22$ \\
\hline 25,27 & $7.39(\mathrm{t}, J=7.3 \mathrm{~Hz})$ & - & 128.4 & - & 1 & + & - & / \\
\hline 26 & $7.33(\mathrm{t}, J=7.2 \mathrm{~Hz})$ & - & 127.9 & - & - & + & - & / \\
\hline 29 & I & I & 155.7 & - & / & / & l & / \\
\hline $30 / 30^{\prime}$ & $7.03(\mathrm{~d}, J=8.4 \mathrm{~Hz})$ & 6.94 & 114.9 & - & - & + & H-31,33 & $\mathrm{H}-10$ \\
\hline $31,33^{a}$ & $7.10(\mathrm{~d}, J=8.3 \mathrm{~Hz})$ & - & 129.8 & - & $\mathrm{C}-29, \mathrm{C}-35$ & + & H-30,34 & H-35 \\
\hline 32 & l & / & 130.1 & - & l & / & l & I \\
\hline 35 & 2.23 & 一 & 20.0 & 一 & C-31,33 & + & 1 & H-31,33 \\
\hline
\end{tabular}

${ }^{a}$ The hydrogen was tentatively assigned because it overlap the peak of $\mathrm{H}-18,20 .{ }^{b}$ Carbon atoms coupled with proton.

\section{2 结果与讨论}

\section{1 目标化合物 7 的结构确证}

\subsection{1 目标化合物 7 的 IR 谱}

由表 2 可知，目标产物在 $3172 \sim 3236 \mathrm{~cm}^{-1}$ 处出现 的中等强度吸收峰为 $\mathrm{N}-\mathrm{H}$ 伸缩振动吸收峰, 在 3034
$3064 \mathrm{~cm}^{-1}$ 处出现的吸收峰为不饱和 $\mathrm{C}-\mathrm{H}$ 伸缩振动吸 收峰, 在 2921 2944 和 $2853 \sim 2873 \mathrm{~cm}^{-1}$ 处出现的吸收 峰为饱和 $\mathrm{C}-\mathrm{H}$ 伸缩振动吸收峰, 在 $1672 \sim 1685 \mathrm{~cm}^{-1}$ 处出现的吸收峰为 $\mathrm{C}=\mathrm{O}$ 的伸缩振动吸收峰, 在 1570 $1576,1484 \sim 1496,1463 \sim 1474 \mathrm{~cm}^{-1}$ 处出现的吸收峰为 苯环骨架振动吸收峰, 在 $1604 \sim 1610 \mathrm{~cm}^{-1}$ 处出现的吸 
表 4 目标化合物 $7 \mathbf{a} \sim 7 \mathbf{j}$ 的 ${ }^{1} \mathrm{H}$ NMR 数据 (DMSO- $\left.d_{6}, 400 / 500 \mathrm{MHz}\right)$

Table $4{ }^{1} \mathrm{H}$ NMR spectral data of target compounds $7 \mathbf{a} \sim 7 \mathbf{j}\left(\mathrm{DMSO}-d_{6}, 400 / 500 \mathrm{MHz}\right)$

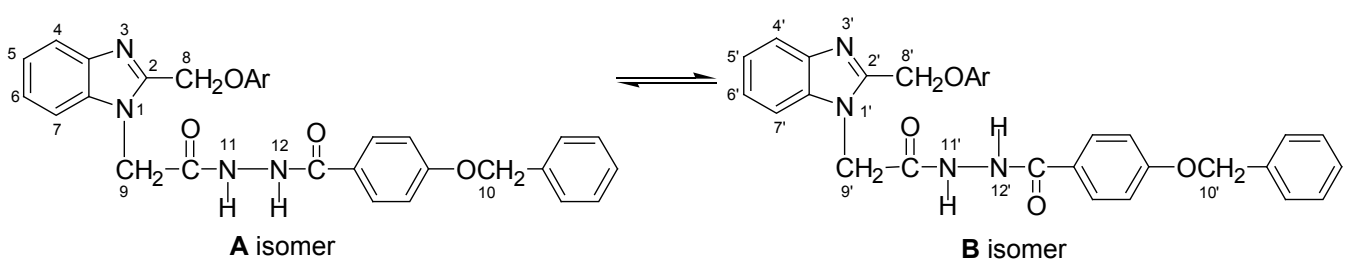

\begin{tabular}{|c|c|c|c|c|c|c|c|c|c|}
\hline \multirow{2}{*}{ Compd. } & \multirow{2}{*}{$\mathrm{Ar}$} & \multicolumn{7}{|c|}{$\delta$} & \multirow{2}{*}{$\begin{array}{l}\text { Isomer } \\
\text { A/B (\%) }\end{array}$} \\
\hline & & $\mathrm{H}-8$ & H-9 & $\mathrm{H}-10$ & $\mathrm{H}-11$ & $\mathrm{H}-12$ & $\mathrm{CH}_{3}$ & $\mathrm{BZM}-\mathrm{H}+\mathrm{ArH}$ & \\
\hline $7 a^{a}$ & $\mathrm{C}_{6} \mathrm{H}_{5}$ & 5.50 & 5.24 & 5.24 & 10.59 & 10.48 & - & 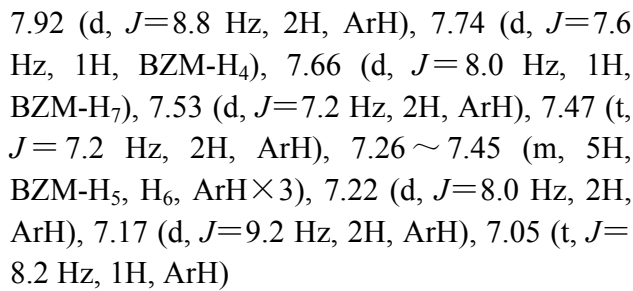 & 80.55 \\
\hline
\end{tabular}

$\begin{array}{lllllllll} & - & - & - & 9.82 & 10.73 & - & & 19.45\end{array}$

7b $\quad 3-\mathrm{CH}_{3} \mathrm{C}_{6} \mathrm{H}_{4} \quad 5.40 \quad 5.16 \quad 5.17 \quad 10.47 \quad 10.39 \quad 2.28 \quad 7.85$ (d, J=8.8 Hz, 2H, ArH), 7.66 (d, $J=8.0$

88.47 $\left.\mathrm{Hz}, 1 \mathrm{H}, \mathrm{BZM}-\mathrm{H}_{4}\right), 7.58(\mathrm{~d}, J=8.1 \mathrm{~Hz}, 1 \mathrm{H}$, BZM-H $\left.{ }_{7}\right), 7.47(\mathrm{~d}, J=7.3 \mathrm{~Hz}, 2 \mathrm{H}, \mathrm{ArH}), 7.39$ (td, $J=6.9,1.6 \mathrm{~Hz}, 2 \mathrm{H}, \mathrm{ArH}), 7.33(\mathrm{t}, J=9.6 \mathrm{~Hz}$, $1 \mathrm{H}, \quad \mathrm{ArH}), 7.30(\mathrm{td}, J=7.7,0.9 \mathrm{~Hz}, 1 \mathrm{H}$, BZM-H 6 ), $7.23(\mathrm{td}, J=7.6,0.8 \mathrm{~Hz}, 1 \mathrm{H}, \mathrm{BZM}-$ $\left.\mathrm{H}_{5}\right), 7.18(\mathrm{t}, J=7.9 \mathrm{~Hz}, 1 \mathrm{H}, \mathrm{ArH}), 7.10(\mathrm{~d}, J=$ $8.9 \mathrm{~Hz}, 2 \mathrm{H}, \mathrm{ArH}), 6.98(\mathrm{~s}, 1 \mathrm{H}, \mathrm{ArH}), 6.94(\mathrm{dd}$, $J=8.2,2.3 \mathrm{~Hz}, 1 \mathrm{H}, \mathrm{ArH}), 6.78(\mathrm{~d}, J=9.0 \mathrm{~Hz}$, $1 \mathrm{H}, \mathrm{ArH})$

$\begin{array}{llllllll} & 5.29 & 5.21 & 5.12 & 9.71 & - & 2.25 & \\ 7 & 4-\mathrm{CH}_{3} \mathrm{C}_{6} \mathrm{H}_{4} & 5.38 & 5.15 & 5.17 & 10.47 & 10.36 & 2.23\end{array} \quad 7.85(\mathrm{~d}, J=8.7 \mathrm{~Hz}, 2 \mathrm{H}, \mathrm{ArH}), 7.65(\mathrm{~d}, J=7.9 \mathrm{~Hz}$, $1 \mathrm{H}, \quad$ BZM- $\left.\mathrm{H}_{4}\right), 7.57(\mathrm{~d}, J=8.0 \mathrm{~Hz}, \quad 1 \mathrm{H}$, BZM-H $\left.H_{7}\right), 7.45$ (d, $\left.J=7.4 \mathrm{~Hz}, 2 \mathrm{H}, \mathrm{ArH}\right), 7.39$ (t, $J=7.3 \mathrm{~Hz}, 2 \mathrm{H}, \mathrm{ArH}), 7.33(\mathrm{t}, J=7.2 \mathrm{~Hz}, 1 \mathrm{H}$, $\mathrm{ArH}), 7.29\left(\mathrm{t}, J=7.7 \mathrm{~Hz}, 1 \mathrm{H}, \mathrm{BZM}-\mathrm{H}_{6}\right), 7.23(\mathrm{t}$, $\left.J=7.4 \mathrm{~Hz}, 1 \mathrm{H}, \mathrm{BZM}-\mathrm{H}_{5}\right), 7.10(\mathrm{~d}, J=8.3 \mathrm{~Hz}$, $4 \mathrm{H}, \mathrm{ArH}), 7.03$ (d, $J=8.4 \mathrm{~Hz}, 2 \mathrm{H}, \mathrm{ArH})$

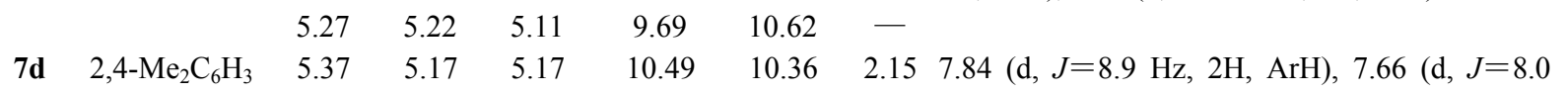

$\left.2.20 \mathrm{~Hz}, 1 \mathrm{H}, \mathrm{BZM}-\mathrm{H}_{4}\right), 7.58(\mathrm{~d}, J=8.1 \mathrm{~Hz}, 1 \mathrm{H}$, BZM-H 7 ), 7.45 (d, $J=7.2 \mathrm{~Hz}, 2 \mathrm{H}, \mathrm{ArH}), 7.39$ (t, $J=7.4 \mathrm{~Hz}, 2 \mathrm{H}, \mathrm{ArH}), 7.33(\mathrm{t}, J=7.3 \mathrm{~Hz}, 1 \mathrm{H}$, ArH), 7.30 (t, $\left.J=7.2 \mathrm{~Hz}, 1 \mathrm{H}, \mathrm{BZM}-\mathrm{H}_{6}\right), 7.24(\mathrm{t}$, $\left.J=7.5 \mathrm{~Hz}, 1 \mathrm{H}, \mathrm{BZM}-\mathrm{H}_{5}\right), 7.10(\mathrm{~d}, J=9.0 \mathrm{~Hz}$, 2H, ArH), 7.08 (d, J=8.4 Hz, 1H, ArH), 6.97 (s, $1 \mathrm{H}, \mathrm{ArH}), 6.95$ (d, $J=8.2 \mathrm{~Hz}, 1 \mathrm{H}, \mathrm{ArH})$

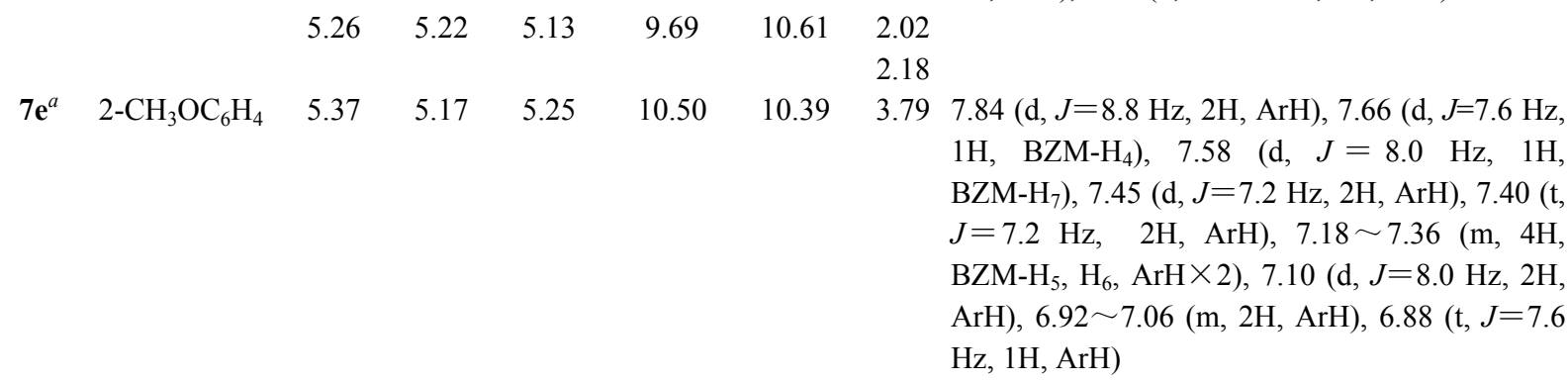




\begin{tabular}{|c|c|c|c|c|c|c|c|c|c|}
\hline \multirow{2}{*}{ No. } & \multirow{2}{*}{$\mathrm{Ar}$} & \multicolumn{7}{|c|}{$\delta$} & \multirow{2}{*}{$\begin{array}{c}\text { Isomer } \\
\mathbf{A} / \mathbf{B}(\%)\end{array}$} \\
\hline & & $\mathrm{H}-8$ & $\mathrm{H}-9$ & $\mathrm{H}-10$ & $\mathrm{H}-11$ & $\mathrm{H}-12$ & $\mathrm{CH}_{3}$ & BZM-H+ArH & \\
\hline \multirow[t]{2}{*}{$7 f^{a}$} & $4-\mathrm{CH}_{3} \mathrm{OC}_{6} \mathrm{H}_{4}$ & 5.36 & 5.16 & 5.17 & 10.50 & 10.40 & 3.69 & $\begin{array}{l}7.85(\mathrm{~d}, J=8.8 \mathrm{~Hz}, 2 \mathrm{H}, \mathrm{ArH}), 7.65(\mathrm{~d}, J=8.0 \mathrm{~Hz}, \\
\left.1 \mathrm{H}, \mathrm{BZM}-\mathrm{H}_{4}\right), 7.58\left(\mathrm{~d}, J=7.6 \mathrm{~Hz}, 1 \mathrm{H}, \mathrm{BZM}-\mathrm{H}_{7}\right), \\
7.46(\mathrm{~d}, J=7.2 \mathrm{~Hz}, 2 \mathrm{H}, \mathrm{ArH}), 7.40(\mathrm{t}, J=7.2 \mathrm{~Hz}, \\
2 \mathrm{H}, \mathrm{ArH}), 7.35(\mathrm{t}, J=6.8 \mathrm{~Hz}, 1 \mathrm{H}, \mathrm{ArH}), 7.29(\mathrm{t}, J \\
\left.=7.8 \mathrm{~Hz}, 1 \mathrm{H}, \mathrm{BZM}-\mathrm{H}_{6}\right), 7.23(\mathrm{t}, J=7.6 \mathrm{~Hz}, 1 \mathrm{H}, \\
\left.\mathrm{BZM}-\mathrm{H}_{5}\right), 7.02 \sim 7.18(\mathrm{~m}, 4 \mathrm{H}, \mathrm{ArH}), 6.87(\mathrm{~d}, J= \\
8.8 \mathrm{~Hz}, 2 \mathrm{H}, \mathrm{ArH})\end{array}$ & 89.90 \\
\hline & & - & - & - & 9.73 & 10.66 & - & & 10.10 \\
\hline \multirow[t]{2}{*}{$7 \mathrm{~g}$} & $4-\mathrm{IC}_{6} \mathrm{H}_{4}$ & 5.43 & 5.15 & 5.18 & 10.47 & 10.36 & - & $\begin{array}{l}7.85(\mathrm{~d}, J=8.9 \mathrm{~Hz}, 2 \mathrm{H}, \mathrm{ArH}), 7.66(\mathrm{~d}, J=8.0 \mathrm{~Hz}, \\
\left.1 \mathrm{H}, \mathrm{BZM}-\mathrm{H}_{4}\right), 7.61(\mathrm{~d}, J=9.0 \mathrm{~Hz}, 2 \mathrm{H}, \mathrm{ArH}), \\
7.58\left(\mathrm{~d}, J=8.2 \mathrm{~Hz}, 1 \mathrm{H}, \mathrm{BZM}-\mathrm{H}_{7}\right), 7.45(J=7.2 \\
\mathrm{Hz}, 2 \mathrm{H}, \mathrm{ArH}), 7.39(\mathrm{t}, J=7.0 \mathrm{~Hz}, 2 \mathrm{H}, \mathrm{ArH}), 7.33 \\
(\mathrm{t}, J=7.2 \mathrm{~Hz}, 1 \mathrm{H}, \mathrm{ArH}), 7.30(\mathrm{td}, J=7.6,0.9 \mathrm{~Hz}, \\
\left.1 \mathrm{H}, \mathrm{BZM}-\mathrm{H}_{6}\right), 7.23(\mathrm{td}, J=7.6,0.9 \mathrm{~Hz}, 1 \mathrm{H}, \\
\left.\mathrm{BZM}-\mathrm{H}_{5}\right), 7.10(\mathrm{~d}, J=8.9 \mathrm{~Hz}, 2 \mathrm{H}, \mathrm{ArH}), 7.00(\mathrm{~d}, \\
J=8.9 \mathrm{~Hz}, 2 \mathrm{H}, \mathrm{ArH})\end{array}$ & 86.46 \\
\hline & & 5.32 & 5.22 & 5.10 & 9.69 & 10.61 & - & & 13.54 \\
\hline \multirow[t]{2}{*}{$7 \mathrm{~h}$} & $4-\mathrm{ClC}_{6} \mathrm{H}_{4}$ & 5.44 & 5.15 & 5.18 & 10.47 & 10.37 & - & $\begin{array}{l}7.85(\mathrm{~d}, J=8.8 \mathrm{~Hz}, 2 \mathrm{H}, \mathrm{ArH}), 7.67(\mathrm{~d}, J=7.9 \mathrm{~Hz}, \\
\left.1 \mathrm{H}, \mathrm{BZM}-\mathrm{H}_{4}\right), 7.58\left(\mathrm{~d}, J=8.1 \mathrm{~Hz}, 1 \mathrm{H}, \mathrm{BZM}-\mathrm{H}_{7}\right), \\
7.45(\mathrm{dd}, J=7.9,1.4,2 \mathrm{H}, \mathrm{ArH}), 7.39(\mathrm{t}, J=7.4 \\
\mathrm{Hz}, 2 \mathrm{H}, \mathrm{ArH}), 7.33 \sim 7.37(\mathrm{~m}, 3 \mathrm{H}, \mathrm{ArH}), 7.30 \\
\left(\mathrm{td}, J=7.7,1.0 \mathrm{~Hz}, 1 \mathrm{H}, \mathrm{BZM}-\mathrm{H}_{6}\right), 7.23(\mathrm{td}, J= \\
\left.7.6,0.9 \mathrm{~Hz}, 1 \mathrm{H}, \mathrm{BZM}-\mathrm{H}_{5}\right), 7.12(\mathrm{~d}, J=9.0 \mathrm{~Hz}, \\
2 \mathrm{H}, \mathrm{ArH}), 7.10(\mathrm{~d}, J=8.9 \mathrm{~Hz}, 2 \mathrm{H}, \mathrm{ArH})\end{array}$ & 88.66 \\
\hline & & 5.33 & 5.20 & 5.11 & 9.70 & - & - & & 11.34 \\
\hline \multirow[t]{2}{*}{$7 i^{a}$} & $1-\mathrm{C}_{10} \mathrm{H}_{7}$ & 5.64 & 5.17 & 5.25 & 10.55 & 10.40 & - & $\begin{array}{l}8.23(\mathrm{~d}, J=8.4 \mathrm{~Hz}, 1 \mathrm{H}, \mathrm{ArH}), 7.89(\mathrm{~d}, J=7.6 \mathrm{~Hz}, \\
1 \mathrm{H}, \mathrm{ArH}), 7.84(\mathrm{~d}, J=8.8 \mathrm{~Hz}, 2 \mathrm{H}, \mathrm{ArH}), 7.70(\mathrm{~d}, \\
\left.J=8.0 \mathrm{~Hz}, 1 \mathrm{H}, \mathrm{BZM}-\mathrm{H}_{4}\right), 7.62(\mathrm{~d}, J=8.4 \mathrm{~Hz}, \\
\left.1 \mathrm{H}, \mathrm{BZM}-\mathrm{H}_{7}\right), 7.20 \sim 7.58(\mathrm{~m}, 12 \mathrm{H}, \mathrm{ArH}), 7.10 \\
(\mathrm{~d}, J=8.8 \mathrm{~Hz}, 2 \mathrm{H}, \mathrm{ArH})\end{array}$ & 83.84 \\
\hline & & 5.53 & 5.22 & 5.20 & 9.75 & - & - & & 16.16 \\
\hline \multirow[t]{2}{*}{$7 \mathbf{j}$} & $2-\mathrm{C}_{10} \mathrm{H}_{7}$ & 5.55 & 5.18 & 5.21 & 10.44 & 10.44 & - & $\begin{array}{l}7.83 \sim 7.88(\mathrm{~m}, 4 \mathrm{H}, \mathrm{ArH}), 7.81(\mathrm{~d}, J=8.2 \mathrm{~Hz}, \\
1 \mathrm{H}, \mathrm{ArH}), 7.68\left(\mathrm{~d}, J=8.0 \mathrm{~Hz}, 1 \mathrm{H}, \mathrm{BZM}-\mathrm{H}_{4}\right), \\
7.57 \sim 7.63\left(\mathrm{~m}, 2 \mathrm{H}, \mathrm{BZM}-\mathrm{H}_{7}, \mathrm{ArH}\right), 7.43 \sim 7.50 \\
(\mathrm{~m}, 3 \mathrm{H}, \mathrm{ArH}), 7.33 \sim 7.42(\mathrm{~m}, 5 \mathrm{H}, \mathrm{ArH}), 7.31(\mathrm{td}, \\
\left.J=7.6,1.0 \mathrm{~Hz}, 1 \mathrm{H}, \mathrm{BZM}-\mathrm{H}_{6}\right), 7.24(\mathrm{td}, J=7.6, \\
\left.0.9 \mathrm{~Hz}, 1 \mathrm{H}, \mathrm{BZM}-\mathrm{H}_{5}\right), 7.10(\mathrm{~d}, J=8.9 \mathrm{~Hz}, 2 \mathrm{H}, \\
\text { ArH })\end{array}$ & 87.05 \\
\hline & & 5.45 & 5.19 & 5.16 & 9.70 & - & - & & 12.95 \\
\hline
\end{tabular}

${ }^{a} 400 \mathrm{MHz}$ 仪器测试.

收峰为 $\mathrm{C}=\mathrm{N}$ 伸缩振动吸收峰, 在 $1504 \sim 1517 \mathrm{~cm}^{-1}$ 处 出现的吸收峰为 $\mathrm{N}-\mathrm{H}$ 弯曲振动吸收峰, 在 1435 1439 和 $1372 \sim 1386 \mathrm{~cm}^{-1}$ 处出现的吸收峰为饱和 $\mathrm{C}-\mathrm{H}$ 的弯 曲振动吸收峰, 在 $1237 \sim 1254$ 和 $1014 \sim 1032 \mathrm{~cm}^{-1}$ 处出 现的吸收峰为 $\mathrm{C}-\mathrm{O}$ 的伸缩振动吸收峰.

\subsection{2 目标化合物 7 的 NMR 谱}

观察目标化合物 $7 \mathbf{a} \sim \mathbf{7 j}$ 的 ${ }^{1} \mathrm{H}$ NMR 谱发现, 高场 $\mathrm{CH}_{2}$ 质子出峰的数目以及低场 $\mathrm{NH}$ 质子出峰的数目均大 于理论的数目, 这说明目标化合物可能存在着两种异构 体, 即 $\mathbf{A}$ 和 $\mathbf{B}$ 异构体. 为了证明这种假设的成立，同时
对目标化合物 7 的 ${ }^{1} \mathrm{H}$ NMR 进行归属, 本文利用 ${ }^{1} \mathrm{H}$ $\mathrm{NMR},{ }^{13} \mathrm{C} \mathrm{NMR}, 2 \mathrm{D} \mathrm{NMR}$ (包括 HMBC, HSQC, ${ }^{1} \mathrm{H}-{ }^{1} \mathrm{H}$ COSY 和 NOESY) 以及变温 ${ }^{1} \mathrm{H}$ NMR 技术对代表化合物 7c 进行了 ${ }^{1} \mathrm{H}$ 和 ${ }^{13} \mathrm{C} \mathrm{NMR}$ 谱归属. 为了便于谱峰的归 属, 将两种异构体的相关原子进行编号(Eq. 1). 代表化 合物 7c 的 ${ }^{1} \mathrm{H}$ NMR, ${ }^{13} \mathrm{C} \mathrm{NMR}, \mathrm{HMBC}, \mathrm{HSQC},{ }^{1} \mathrm{H}-{ }^{1} \mathrm{H}$ COSY, NOESY 和变温实验的 ${ }^{1} \mathrm{H}$ NMR 谱见图 $1 \sim 7$.

根据 7c 的 ${ }^{1} \mathrm{H}$ NMR 可知(图 1), 高场 $\delta_{\mathrm{H}} 2.23$ 可直接 归属为 $\mathrm{H}-35$, 根据电子云密度可知, H-10 与 $\mathrm{H}-11$ 和 $\mathrm{H}-22$ 相比应在较低场, 所以 $\delta_{\mathrm{H}} 5.38$ 可直接归属为 H-10. 
<smiles>[14CH4]</smiles>

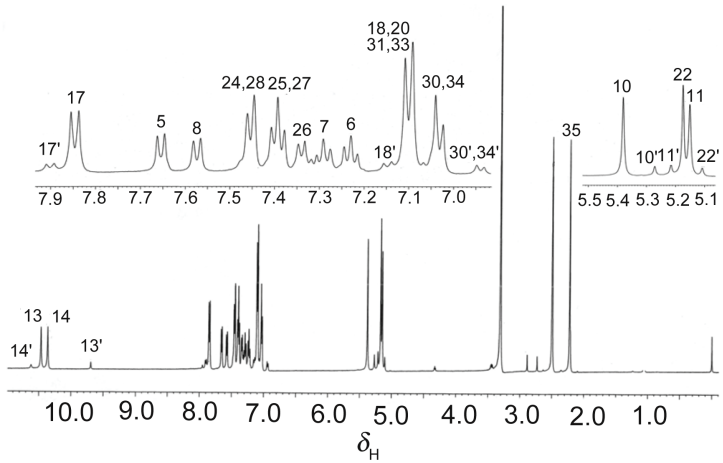

图 1 化合物 $7 \mathbf{c}$ 的 ${ }^{1} \mathrm{H} \mathrm{NMR}$ 谱

Figure $1{ }^{1} \mathrm{H}$ NMR spectrum of compound $7 \mathrm{c}$

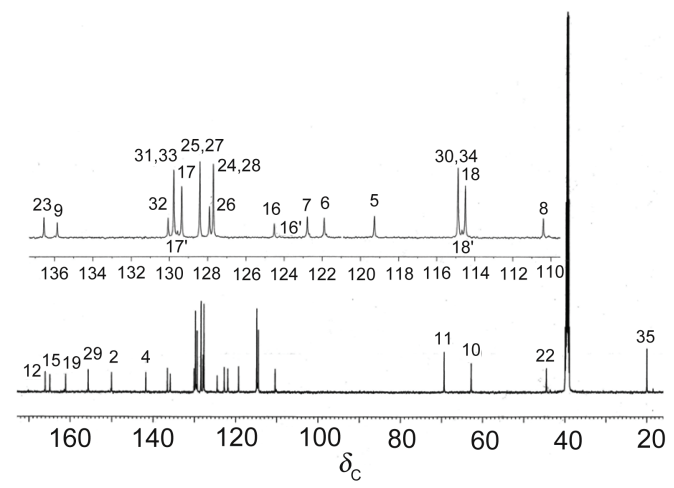

图 2 化合物 $7 \mathbf{c}$ 的 ${ }^{13} \mathrm{C} \mathrm{NMR}$ 谱

Figure $2{ }^{13} \mathrm{C}$ NMR spectrum of compound $7 \mathrm{c}$

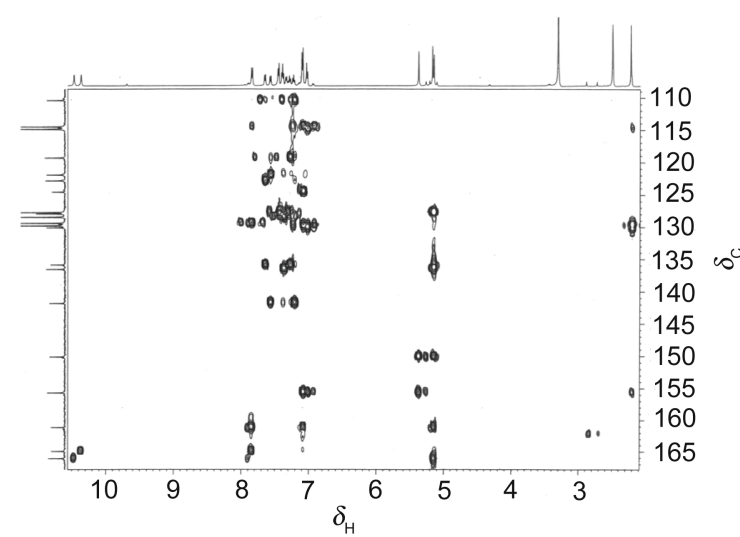

图 3 化合物 7c 的 HMBC 谱

Figure 3 HMBC spectrum of compound $7 \mathbf{c}$

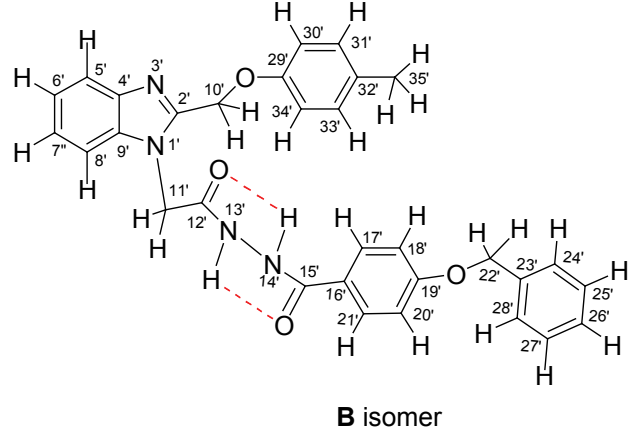

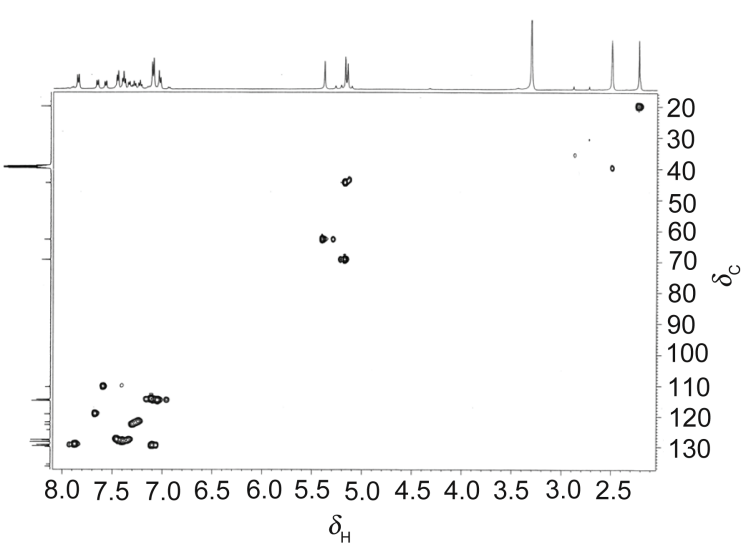

图 4 化合物 7c 的 HSQC 谱

Figure 4 HSQC spectrum of compound 7c

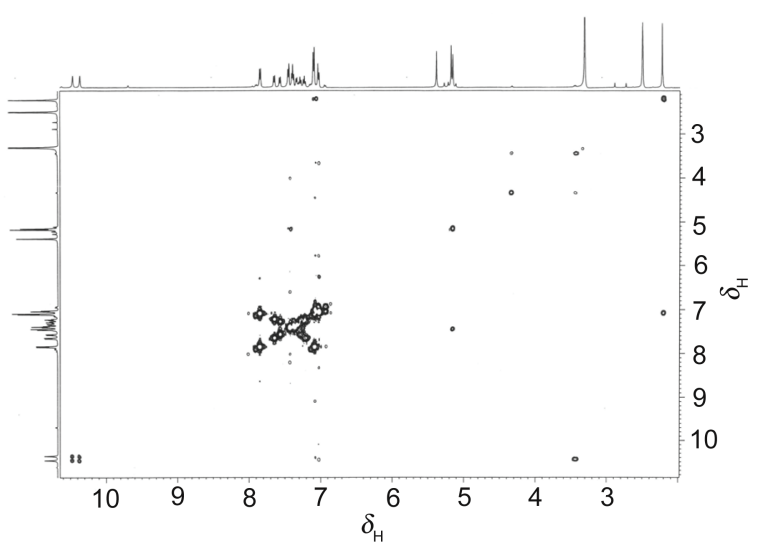

图 5 化合物 7c 的 ${ }^{1} \mathrm{H}-{ }^{1} \mathrm{HCOSY}$ 谱

Figure $5{ }^{1} \mathrm{H}-{ }^{1} \mathrm{H}$ COSY spectrum of compound $7 \mathbf{c}$

由 $\mathrm{HMBC}$ 谱(图 3)可知, $\mathrm{H}-10 \delta_{\mathrm{H}} 5.38$ 和 $\delta_{\mathrm{H}} 5.27$ 与 $\delta_{\mathrm{C}}$ $150.1, \delta_{\mathrm{C}} 150.1$ 与 $\delta_{\mathrm{H}} 5.15$ 存在着长程相关, 则 $\delta_{\mathrm{C}} 150.1$ 归属为 C-2, $\delta_{\mathrm{H}} 5.27$ 归属为 H-10', $\delta_{\mathrm{H}} 5.15$ 归属为 H-11. 根据 HSQC 谱(图 4), 即可分别归属出 C-10 ( $\left.\delta_{\mathrm{C}} 62.8\right)$, C-11 $\left(\delta_{\mathrm{C}} 69.4\right)$ 和 C-35 ( $\left.\delta_{\mathrm{C}} 20.0\right)$. 因在 HSQC 谱中, C-11 $\left(\delta_{\mathrm{C}} 69.4\right)$ 与 $\mathrm{H}-11$ 和 $\delta_{\mathrm{H}} 5.22$ 存在着交叉峰, 所以 $\delta_{\mathrm{H}} 5.22$ 为 H-11'. 高场剩余的信号峰 $\delta_{\mathrm{H}} 5.17$ 和 $\delta_{\mathrm{H}} 5.11$, 则分别 归属为 H-22 和 H-22'. 再根据 HSQC 谱, 相应的 C-22 $\left(\delta_{\mathrm{C}}\right.$ 44.6) 和 C-22' $\left(\delta_{\mathrm{C}} 43.6\right)$ 即可确定. 至此, 高场的三个 $\mathrm{CH}_{2}$ 和 $\mathrm{CH}_{3}$ 的质子及碳信号得到了归属. 


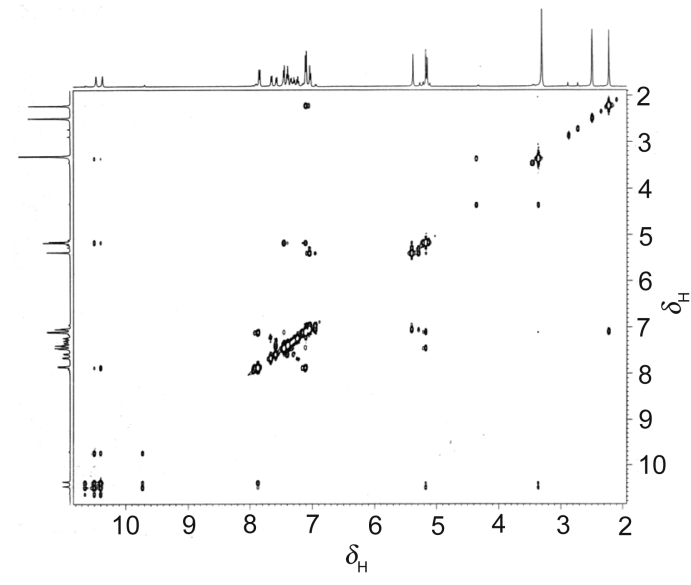

图 6 化合物 7c 的 NOESY 谱

Figure 6 NOESY spectrum of compound 7c

A 环的归属: 由 $\mathrm{HMBC}$ 谱可知, C-35 与 $\delta_{\mathrm{H}} 7.10$ $(\mathrm{H}-31,33), \mathrm{H}-35$ 与 $\delta_{\mathrm{C}} 129.8(\mathrm{C}-31,33)$ 和 $\delta_{\mathrm{C}} 130.1(\mathrm{C}-32)$, $\mathrm{H}-10$ 和 $\mathrm{H}-10^{\prime}$ 与 $\mathrm{C}-2\left(\delta_{\mathrm{C}} 150.1\right)$ 和 $\delta_{\mathrm{C}} 155.7$ (C-29) 存在着 远程耦合. 在 ${ }^{1} \mathrm{H}-{ }^{1} \mathrm{H}$ COSY 谱中(图 5), H-31,33 与 $\delta_{\mathrm{H}} 7.03$ $(\mathrm{H}-30,34)$ 存在交叉峰，由 HSQC 谱即可确定出 C-30,34 $\left(\delta_{\mathrm{C}} 114.9\right)$, 而 C-30,34 又与 $\delta_{\mathrm{H}} 6.94$ 存在交叉峰, 所以 $\delta_{\mathrm{H}}$ 6.94 归属为 $\mathrm{H}-30^{\prime}, 34$ '.

B 环及低场羰基碳和 $\mathrm{NH}$ 质子的归属: 由 $\mathrm{HMBC}$ 谱 可知, $\mathrm{H}-11$ 与 $\delta_{\mathrm{C}} 166.1, \delta_{\mathrm{C}} 166.1$ 与 $\delta_{\mathrm{H}} 10.47$ 存在着远程 耦合相关峰, 所以 $\delta_{\mathrm{C}} 166.1$ 归属为 C-12, $\delta_{\mathrm{H}} 10.47$ 归属为 $\mathrm{H}-13$. 通过 ${ }^{1} \mathrm{H}-{ }^{1} \mathrm{H}$ COSY 谱可看出, $\mathrm{H}-13$ 与 $\delta_{\mathrm{H}} 10.36$ 存 在着交叉峰, 则 $\delta_{\mathrm{H}} 10.36$ 为 $\mathrm{H}-14$. 在 $\mathrm{HMBC}$ 谱中, $\mathrm{H}-14$ 与 $\delta_{\mathrm{C}} 164.9, \delta_{\mathrm{C}} 164.9$ 与 $\delta_{\mathrm{H}} 7.85$ 存在长程相关, 所以 $\delta_{\mathrm{C}}$ 164.9 为 $\mathrm{C}-15, \delta_{\mathrm{H}} 7.85$ 为 $\mathrm{H}-17,21$. 又因 $\mathrm{H}-17,21 / \delta_{\mathrm{H}} 7.90$ 与 $\delta_{\mathrm{C}} 161.1$ 也存在长程相关, 所以 $\delta_{\mathrm{H}} 7.90$ 为 $\mathrm{H}-17^{\prime}, 21^{\prime}, \delta_{\mathrm{C}}$ 161.1 为 C-19. 再根据 ${ }^{1} \mathrm{H}-{ }^{1} \mathrm{H}$ COSY 谱和 HSQC 谱, 可确 定出 $\mathrm{H}-18,20\left(\delta_{\mathrm{H}} 7.10\right) / \mathrm{H}-18^{\prime}, 20^{\prime}\left(\delta_{\mathrm{H}} 7.15\right)$ 和 $\mathrm{C}-18,20$
$\left(\delta_{\mathrm{C}} \quad 114.5\right) / \mathrm{C}-18^{\prime}, 20^{\prime} \quad\left(\delta_{\mathrm{C}} 114.7\right), \quad \mathrm{C}-17,21\left(\delta_{\mathrm{C}} 129.4\right) / \mathrm{C}-$ $17^{\prime}, 21^{\prime}\left(\delta_{\mathrm{C}}\right.$ 129.6). 在 HMBC 谱中, 因 $\mathrm{H}-18,20 / \mathrm{H}-18^{\prime}, 20^{\prime}$ 与 $\delta_{\mathrm{C}} 124.5 / \delta_{\mathrm{C}} 124.3$ 存在长程相关, 所以 $\delta_{\mathrm{C}} 124.5 / \delta_{\mathrm{C}}$ 124.3 归属为 C-16/C-16'.

苯并咪唑环的归属: 由 $\mathrm{HMBC}$ 谱可知, $\mathrm{H}-11$ 与 $\mathrm{C}-2$ 和 $\delta_{\mathrm{C}} 135.9$ 也存在着远程耦合, 所以 $\delta_{\mathrm{C}} 135.9$ 被归属为 C-9. 此外, C-9 与 $\delta_{\mathrm{H}} 7.65$ (双峰)和 $\delta_{\mathrm{H}} 7.29$ (三重峰)存在着 长程相关, 则 $\delta_{\mathrm{H}} 7.65$ 为 $\mathrm{H}-5, \delta_{\mathrm{H}} 7.29$ 为 $\mathrm{H}-7$. 再根据 ${ }^{1} \mathrm{H}-{ }^{1} \mathrm{H}$ COSY 谱和 HSQC 谱, 可确定出 H-6 ( $\left.\delta_{\mathrm{H}} 7.23\right)$ 和 $\mathrm{H}-8\left(\delta_{\mathrm{H}} 7.57\right)$ 以及 C-5 $\left(\delta_{\mathrm{C}} 119.3\right), \mathrm{C}-6\left(\delta_{\mathrm{C}} 121.9\right), \mathrm{C}-7\left(\delta_{\mathrm{C}}\right.$ $122.8)$ 和 C-8 $\left(\delta_{\mathrm{C}} 110.4\right)$. 最后, 由 $\mathrm{HMBC}$ 谱可知, H-6 与 $\delta_{\mathrm{C}} 141.8$ 存在着长程相关, 则 $\delta_{\mathrm{C}} 141.8$ 被归属为 C-4.

$\mathrm{C}$ 环的归属: 由 $\mathrm{HMBC}$ 谱可知, $\mathrm{H}-22$ 与 $\mathrm{C}-19\left(\delta_{\mathrm{C}}\right.$ $161.1), \delta_{\mathrm{C}} 136.6$ 和 $\delta_{\mathrm{C}} 127.7$ 存在着远程耦合，则 $\delta_{\mathrm{C}} 136.6$ 为 $\mathrm{C}-23, \delta_{\mathrm{C}} 127.7$ 为 $\mathrm{C}-24,28$. 由 $\mathrm{HSQC}$ 谱即可确定出 $\mathrm{H}-24,28\left(\delta_{\mathrm{H}} 7.45\right)$. 在 $\mathrm{HMBC}$ 谱中, $\mathrm{C}-23\left(\delta_{\mathrm{C}} 136.6\right)$ 与 $\delta_{\mathrm{H}}$ 7.39 存在相关峰, 则 $\delta_{\mathrm{H}} 7.39$ 为 $\mathrm{H}-25,27$. 根据 HSQC 谱, 即可确定 C-25,27 ( $\left.\delta_{\mathrm{C}} 128.4\right)$. 最后, ${ }^{13} \mathrm{C}$ NMR 谱中剩余 信号峰 $\delta_{\mathrm{C}} 127.9$ 被归属为 C-26, 并通过 HSQC 谱可确定 出 H-26 $\left(\delta_{\mathrm{H}} 7.33\right)$.

由 NOESY 谱(图 6)可看出: H-14 与 H-13, H-13 与 $\mathrm{H}-11, \mathrm{H}-14$ 与 $\mathrm{H}-21$ 存在着交叉峰，说明 $\mathrm{H}-11, \mathrm{H}-13$, $\mathrm{H}-14$ 和 $\mathrm{H}-21$ 空间上距离较近并且在同一侧, $\mathrm{N}-\mathrm{H}$ 与 $\mathrm{C}=\mathrm{O}$ 处于反式构型. 所以在室温下, DMSO 中, $\mathrm{A}$ 结构 为主要异构体. 而 H-14'与 $\mathrm{H}-13^{\prime}$ 无相关峰, 则 B 结构为 次要异构体(即全反式构型).

此外，还观察到 H-35 与 H-31,33, H-22 与 H- 18,20 和 H-24,28, H-10 与 H-30,34 也存在着相关峰, 说明它们 空间上也是彼此靠近的. 由此可以得到 A 结构的空间构 型(Eq. 1).

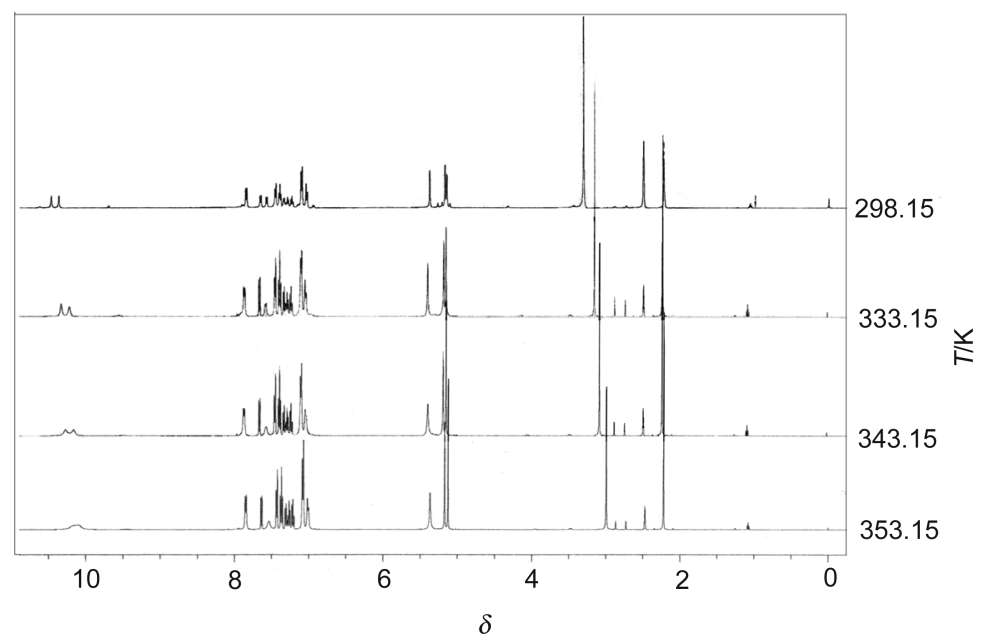

图 7 化合物 7c 在 DMSO- $d_{6}$ 中不同温度的 ${ }^{1} \mathrm{H}$ NMR 谱

Figure $7{ }^{1} \mathrm{H}$ NMR spectra of compound $7 \mathbf{c}$ at various temperature in DMSO- $d_{6}$ 
因为 $\mathrm{C}-12\left(\delta_{\mathrm{C}} 166.1\right)$ 较 C-15 $\left(\delta_{\mathrm{C}} 164.9\right)$ 在低场, 所以 在 B 结构中也应如此. 由 B 结构可以看出, H-13'和 H-14' 均能与 $\mathrm{C}=\mathrm{O}$ 中的氧形成五元环的分子内氢键. 所以 $\mathrm{H}-14$ '较 H-13'在低场. 因此 $\delta_{\mathrm{H}} 10.62$ 归属为 H-14', $\delta_{\mathrm{H}} 9.69$ 归属为 H-13'。

由变温实验(图 7)结果可知: 当温度升高时, $\mathbf{B}$ 异构 体逐渐转化为 $\mathrm{A}$ 异构体, 当温度达到 $60{ }^{\circ} \mathrm{C}$ 时, 在 $\delta_{\mathrm{H}}$ 9.5 11.0 和 $\delta_{\mathrm{H}} 5.0 \sim 5.5$ 处, B 异构体的峰完全消失. 说 明在此温度 $\mathbf{B}$ 异构体已完全转化为 $\mathbf{A}$ 异构体. 同时, 由 于低场的峰明显向高场移动, 说明高温时 $\left(60{ }^{\circ} \mathrm{C}\right)$ 分子 内氢键被破坏, 致使 $\mathrm{N}-\mathrm{N}$ 键能够旋转, B 异构体转化为 A 异构体.

根据 H-10, H-13, H-14, H-17 和 H-30,34 的峰面积的 平均值, 可求出化合物 $7 \mathrm{c}$ 两种异构体 $\mathrm{A}$ 和 $\mathrm{B}$ 的含量, 分 别为 $87.65 \%$ 和 $12.35 \%$.

至此，化合物 $7 \mathbf{c}$ 的两种异构体 (A 和 B) 的 ${ }^{1} \mathrm{H}$ 和 ${ }^{13} \mathrm{C}$ 信号得到了全部归属(表 3 ), 并给出了两种异构体的含 量及空间构型(Eq. 1).

根据化合物 $7 \mathbf{c}$ 的 NMR 谱分析结果, 目标化合物 $7 \mathbf{a} \sim 7 \mathbf{j}$ 的氢谱得以归属(表 4).

\section{References}

[1] Demirayak, S.; Kayagil, I.; Yurttas, L. Eur. J. Med. Chem. 2011, 46, 411.

[2] Woo, H. B.; Eom, Y. W.; Park, K.-S.; Ham, J.; Ahn, C. M.; Lee, S. Bioorg. Med. Chem. Lett. 2012, 22, 933.

[3] Li, Y.-J.; Liu, L.-J.; Jin, K.; Sun, S.-Q.; Xu, Y.-T. Acta Chim. Sinica
2010, 68(16), 1577 (in Chinese). (李英俊, 刘丽军, 靳焜, 孙淑琴, 许永廷, 化学学报, 2010, 68(16), 1577.)

[4] Kaushik, D.; Khan, S. A.; Chawla, G. Med. Chem. Res. 2012, 21, 459.

[5] Soni, L. K.; Narsinghani, T.; Sethi, A. Med Chem Res. 2012, 21, 4330.

[6] Hernández-Luis, F.; Hernández-Campos, A.; Castillo, R.; Navarrete-Vázquez, G.; Soria-Arteche, O.; Hernández-Hernández, M.; Yépez-Muli, L. Eur. J. Med. Chem. 2010, 45, 3135.

[7] Schmidt, S.; Preu, L.; Lemcke, T.; Totzke, F.; Schächtele, C.; Kubbutat, M. H. G.; Kunick, C. Eur. J. Med. Chem. 2011, 46, 2759.

[8] Hearn, M. J.; Cynamon, M. H. Drug Des. Discovery 2003, 18, 103.

[9] Thomas, K. D.; Adhikari, A. V.; Telkar, S.; Telkar, S.; Chowdhury, I. H.; Mahmood, R.; Pal, N. K.; Row, G.; Sumesh, E. Eur. J. Med. Chem. 2011, 46, 5283.

[10] Kovganko, N. V.; Ananich, S. K. Russ. J. Bioorg. Chem. 2004, 30 , 505.

[11] Parra, M.; Hidalgo, P.; Barberá, J.; Carrasco, E.; Saavedra, C. Liq. Cryst. 2006, 33, 391.

[12] Li, Y.-J.; Liu, L.-J.; Jin, K.; Wang, W.; Zhao, N.; Li, L.-N.; Li, C.-Y. Chin. J. Org. Chem. 2009, 29(11), 1825 (in Chinese). (李英俊, 刘丽军, 靳焜, 王巍, 赵楠, 李丽娜, 李春燕, 有机化 学, 2009, 29(11), 1825.)

[13] Li, L.-N. M.S. Thesis, Liaoning Normal University, Dalian, 2011 (in Chinese).

(李丽娜, 硕士论文, 辽宁师范大学, 大连, 2011.)

[14] Cativiela, C.; Serrano, J. L.; Zurbano, M. M. J. Org. Chem. 1995, 60, 3074.

[15] Li, Y.-J.; Ding, W.-G.; Jin, K.; Sun, S.-Q.; Liu, J.; Liu, L.-J.; Wang, J.-K. Chin. J. Org. Chem. 2009, 29(1), 82 (in Chinese). (李英俊, 丁万刚, 靳焜, 孙淑琴, 刘军, 刘丽军, 王金奎, 有机 化学, 2009, 29(1), 82.)

(Cheng, F.) 